\title{
Gravitational Force Explanation Based on Universe as a Superconductor of Matter
}

\section{Sergio P. F.}

Sergio Pérez Felipe, Independent Researcher, Graduated from Computer Science, Madrid, Spain

Email address:

sergiopf@gmail.com

\section{To cite this article:}

Sergio P. F. Gravitational Force Explanation Based on Universe as a Superconductor of Matter. International Journal of Applied Mathematics and Theoretical Physics. Vol. 3, No. 2, 2017, pp. 38-42. doi: 10.11648/j.ijamtp.20170302.13

Received: December 14, 2016; Accepted: March 1, 2017; Published: March 30, 2017

\begin{abstract}
Gravity explained by a new theory, 'Superconducting String Theory', inspired on initial string theories and completely opposite from actual fields based. Strengths are decomposed to make strings behave as one-dimensional with universe acting as a superconductor where resistance is near 0 and matter moves inside. Strong nuclear force, with an attraction of 10.000 Newtons is which makes space to curve, generating acceleration. More matter more acceleration. Electromagnetic moves in 8 decimals, gravity is moved from 3 to more than 30 decimals to work as a superconductor.
\end{abstract}

Keywords: Gravity, Unified Field Theory, Dark Matter, Relativity, Strong Nuclear Force, Gluon, String Theory

\section{Introduction}

The 'Theory of Everything' is a hypothetical theory of physics that explains and connects all known physical phenomena into one. There is a possible solution to the origin of gravity force, postulating it as angular piece of this theory, this solution erases gravity as one of the fundamental forces of nature and unifies it with strong nuclear force.

Let's analyze the forces that occur in the universe transforming string theory. It allows to explain many physical behaviors that without its existence would be practically impossible to understand, even so, these strings have not been able to be discovered and are only that, a theory that serves as an important support to the world of physics. One of the best known theoretical applications about them is how their vibration can provoke the creation of matter, but this is not about theories already written, we are going to place these strings in a simpler way to answer some doubts in subatomic world.

This theory uses 4 dimensions in space and a behavior as one dimension in strings with superconducting capacities. Like an elastic band between V-shaped sticks where the elastic band slides down, the strong nuclear force, forces these strings to bend to fall dawn.

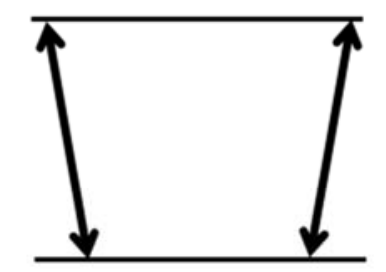

2 strings separated by $180 \mathrm{px}$ $=1$ space unit example

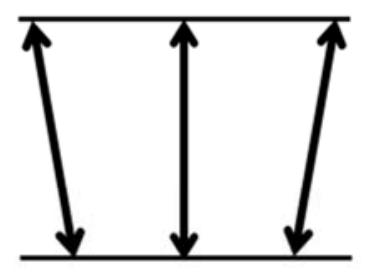

3 strings separated by $90 \mathrm{px}$ $=2$ space units example
Figure 1. String example.

It's not directly related to electromagnetism.

\section{Actors}

\subsection{String Theory}

String theory is a theoretical framework in which the point-like particles of particle physics are replaced by onedimensional objects called strings. Each string that we cross would be the minimum distance that can be traversed during a displacement.

We can note two important qualities of strings:

- Distance to the most distant object detected by the human being is more than 30 billion light years, that means there are beams of light which are able to travel that distance without decreasing its speed (they modify only its wavelength). Like light, an object can move 
into space for a practically unlimited period, as long as it doesn't find a force to stop it. If strings exist, they act as a superconductor of matter with a resistance near 0 .

- In order to generate waves it's easier into a strongly linked structure. Gravitational waves behave like ocean waves which are similar to an uptight net, these tensions can be decomposed as one-dimensional structure for its study. Strings, at same time, could be one or zero-dimensional, like points under extreme bound forces, think about them as something tenser than any cable that holds the heaviest bridge in the world.

The new framework we have drawn would be a set of extremely tense strings, with a practically infinite matter conduction capacity. Remember we are moving into universe at a stimated speed of $600 \mathrm{~km} / \mathrm{sc}$.

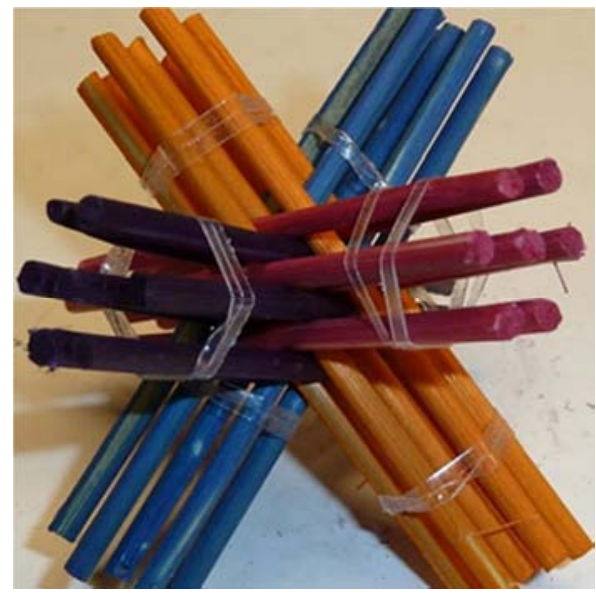

Figure 2. Example of quantum entanglement. Gravity waves behave as an uptight net.

\subsection{Strong Nuclear Force}

Strong nuclear force is another variable. This force allows the atomic nucleus to remain together, being the strongest of the so-called fundamental interactions (gravitational, electromagnetic, strong nuclear, and weak nuclear). Gluon is in charge of this interaction, it has a scope not greater than 10 to the power of -15 meters, preventing matter to separate by a constant attraction force between quarks of maximum 10.000 $\mathrm{N}(\mathrm{F})$.

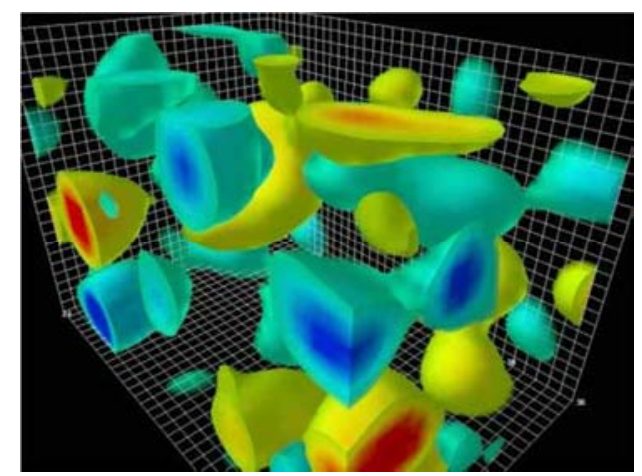

Figure 3. Gluon into vacuum. (Special Research Centre for the Subatomic Structure of Matter).
This real picture illustrates the three-dimensional structure of gluon-field configurations, describing the vacuum properties. The volume of the box is 2,4 by 2,4 by $3,6 \mathrm{fm}$. Contrary to the concept of an empty vacuum, this induces chromo-electric and chromo-magnetic fields in its lowest energy state. The frame rate into this example is billions of billions frames per second (FPS).

\section{Superconducting String Theory (SST)}

\subsection{Fundamentals}

We have created a scenario with a superconductor of matter interacting with a force that makes that matter hold together, but, how can they interact with each other? The most simple is to think about two V-shaped sticks (simulating the strings), and an elastic band that tight them at the most opened side (it would simulate the gluon, with size 10 to the power of -15 meters). If sticks are sufficiently lubricated and tense, what does the elastic band do? It will slide to the thinnest side. More elastic bands, more force will be exerted on the sticks to join them, so next bands will slide even faster (equally, more mass causes more attraction).

We are talking about unknown limits in known world, such as infinite conduction or tensions never seen in materials.

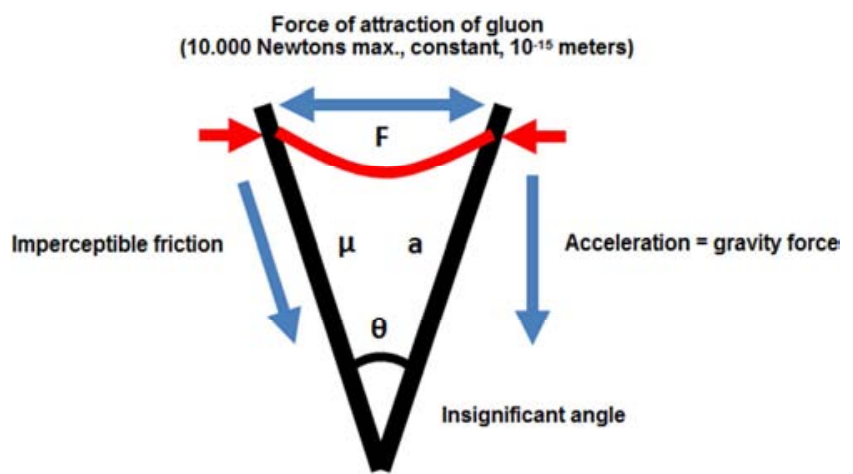

Figure 4. Implied forces.

Suddenly, we have erased one of the fundamental forces of nature, gravity force doesn't really exist, exists the strong nuclear force interacting with strings. I have called this theory 'Superconducting String Theory (SST)'.

\subsection{Calculations}

Apply formulas from inclined planes (Newton's second law). Simulation is in horizontal direction.

Friction is imperceptible and acceleration down the plane is matched with gravity acceleration in our planet. Vertical force is not gravity force, it is gluon force, which values ares estimated, so we keep force $10.000 \mathrm{~N}\left(\mathrm{~F}_{1}\right)$ and mass 0,0002 $\mathrm{eV} / \mathrm{c}^{2}\left(\mathrm{~m}_{2}\right)$. It can be considered vertical angle, but it's depreciable. 


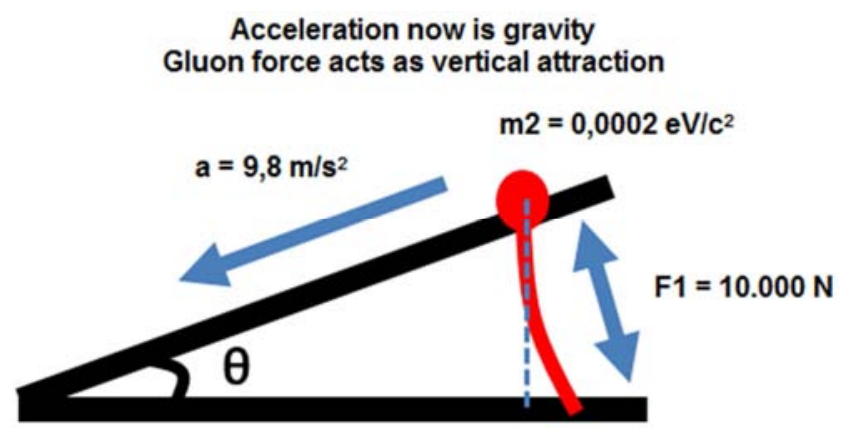

Figure 5. Inclined plane forces (gluon catching strings).

$$
m_{1} \times g \times \operatorname{sen}(x)=m_{2} \times a
$$

Match vertical force $\left(\mathrm{F}_{1}\right)$ with the attraction force from one gluon (estimated max. constant $10.000 \mathrm{~N}$ ).

$$
\begin{aligned}
& F_{l}=m_{1} \times g=10.000 \mathrm{~N} \\
& F_{1} \times \operatorname{sen}(x)=m_{2} \times a
\end{aligned}
$$

Convert variables to metric system considering the mass of the gluon $\left(0,0002 \mathrm{eV} / \mathrm{c}^{2}\right)$.

$$
\begin{gathered}
1 \mathrm{eV} / \mathrm{c}^{2}=1,782662 \times 10^{-36} \mathrm{~kg} \\
m_{2}=0,0002 \times 1,782662 \times 10^{-36}=3,565324 \times 10^{-40} \mathrm{~kg} \\
a=9,8 \mathrm{~m} / \mathrm{s}^{2} \\
F_{2}=m_{2} \times a=3,565324 \times 10^{-40} \times 9,8=3,49401752 \times 10^{-39} \mathrm{~N}
\end{gathered}
$$

Angle calculation of all strings under gluon influence.

$$
\begin{gathered}
x=\operatorname{arcsen}\left(F_{2} / F_{1}\right) \\
F_{2} / F_{1}=3,49401752 \times 10^{-43} \mathrm{~N} \\
x=\operatorname{arcsen}\left(3,49401752 \times 10^{-43}\right)=3,49401752 \times 10^{-43}
\end{gathered}
$$

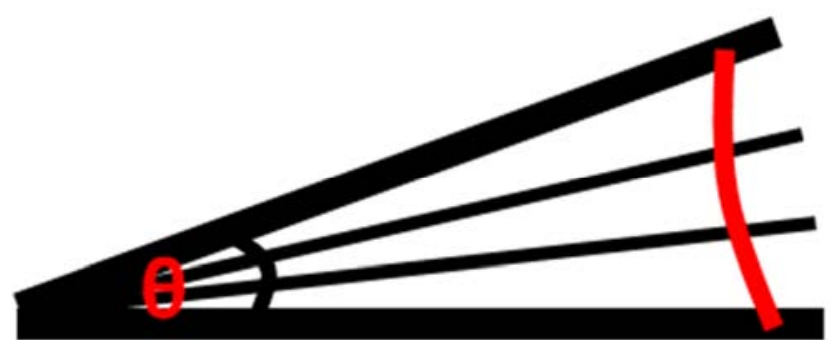

Figure 6. $\theta$ angle of all strings gluon can catch.

\section{Conclusions}

It can explain:

- What is gravity force.

- Unified field theory between gravity and strong nuclear force.

- New behavior in dark matter because of differences in density of the superconductor. Lower density generates bigger angle, this implies bigger attraction force. If threads are separated, matter $\left(\mathrm{m}_{2}\right)$ becomes energy $\left(\mathrm{F}_{1}\right)$. Some places at universe could have bigger accelerations because of this effect; this means much less dark matter (which is stimated at approximately $27 \%$ of the mass and energy in the observable universe).

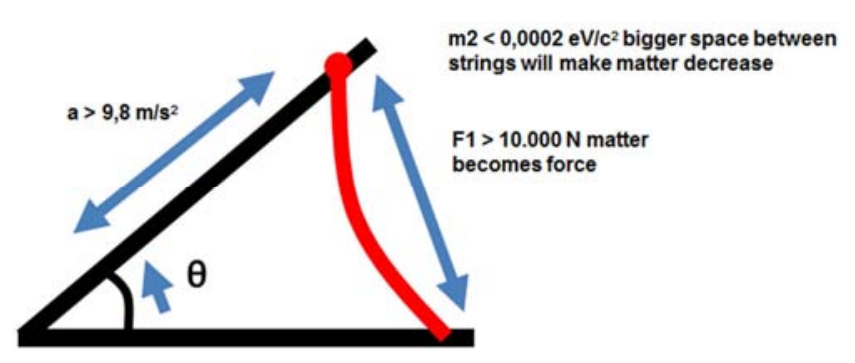

Figure 7. Increase the separation, increase the force exerted. Matter is transformed into energy.

$$
\begin{gathered}
F_{1} \times \operatorname{sen}(x)=m_{2} \times a \\
1 e V / c^{2}=1,782662 \times 10^{-36} \mathrm{~kg} \\
m_{2}=0,0002 \times 1,782662 \times 10^{-36}=3,565324 \times 10^{-40} \mathrm{~kg} \\
F_{2}=m_{2} \times a=3,565324 \times 10^{-40} \times a\left(\mathrm{~kg} /\left(\mathrm{m} / \mathrm{s}^{2}\right)\right)
\end{gathered}
$$

Calculate acceleration in relation to angle.

$$
x=\operatorname{arcsen}\left(F_{2} / F_{l}\right)
$$

$F_{2} / F_{I}=\left(3,565324 \times 10^{-40} \times a\right) / 10.000=\left(3,565324 \times 10^{-44} \times a\right)$ $x=\operatorname{arcsen}\left(3,565324 \times 10^{-44} \times a\right)=\left(3,565324 \times 10^{-44} \times a\right)^{\circ}$

Bigger angle generates more acceleration.

$$
a=\left(x / 3,565324 \times 10^{-44}\right) \mathrm{m} / \mathrm{sg}^{2}
$$

- Einstein field equations. Apply Hooke's law to gluon force as a vertical spring to calculate tensions.

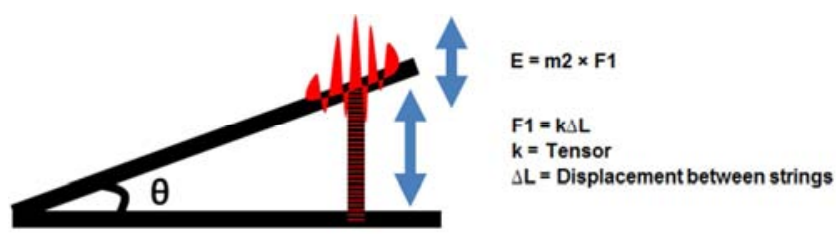

Figure 8. Energy don't disappear becomes matter.

Gluon force has negative correlation when transforming into matter, decrease $F_{1}$ or increase $m_{2}$ implies less acceleration.

$$
a=F_{1} \times \operatorname{sen}(x) / m_{2}
$$

$$
a=5.000 \times \operatorname{sen}\left(3,49401752 \times 10^{-43}\right) / 3,565324 \times 10^{-40}
$$

$$
a=4,9 \mathrm{~m} / \mathrm{s}^{2}
$$

- Spherical and circular movements at planets and galaxies. Space deforms not proportionally to create more acceleration near accumulation of matter, behaving like an elastic material; this behavior can be quantified by Young's modulus which represents the factor of proportionality in Hooke's law at non-linear systems. 

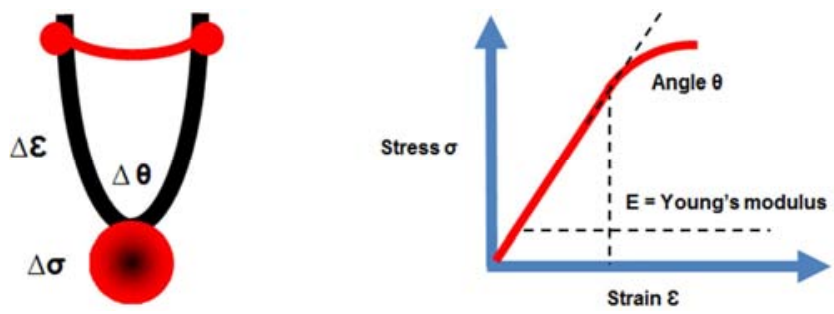

Figure 9. Angle originated by big amounts of matter has an interaction over very large distances.

The elastic modulus or Young's modulus (E) depends on the force exerted by matter $(\sigma)$ and the deformation at each point of the resulting vector $(\mathcal{E})$.

$$
E=\Delta \sigma / \Delta \varepsilon
$$

Force exerted by the angle $(\mathrm{F} \theta)$, increases $(\Delta)$ faster than force exerted by gluon $\left(\mathrm{F}_{1}\right)$ and its relation with matter $\left(\mathrm{m}_{2}\right)$.

$$
\Delta F \theta>\Delta F_{1} / \Delta m_{2}
$$

This gluon-matter relation also modifies the density of the superconductor in space, since it induces their approach, therefore we can speak of the existence of a bulk modulus $(\mathrm{K})$.

$$
K=-V(\Delta p / \Delta V)
$$

The bulk modulus $(\mathrm{K})$ depends on pressure changes $(\mathrm{p})$ and volume $(\mathrm{V})$.

Other properties as volume viscosity also called bulk viscosity can be applied.

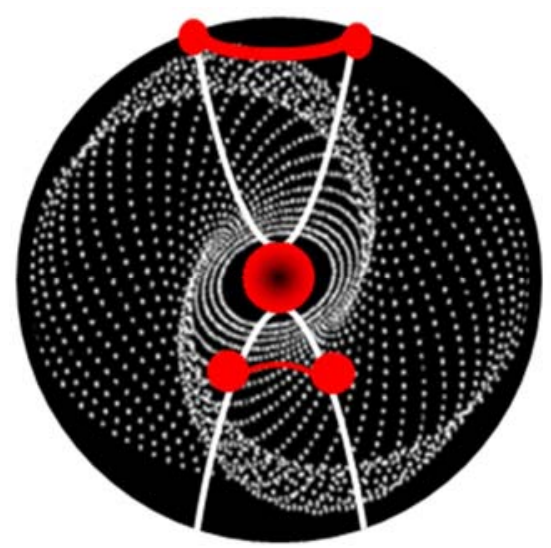

Figure 10. All these variables help to create shapes in galaxies like the golden spiral $(\varphi=1,6180)$.

- Dark energy and universe's expansion. The behaviuor of the strings implies to have any kind of polaritation to expand, at least, strong enough to avoid get closer and restablish its structure after any contraction. This strength propagates over long distances.

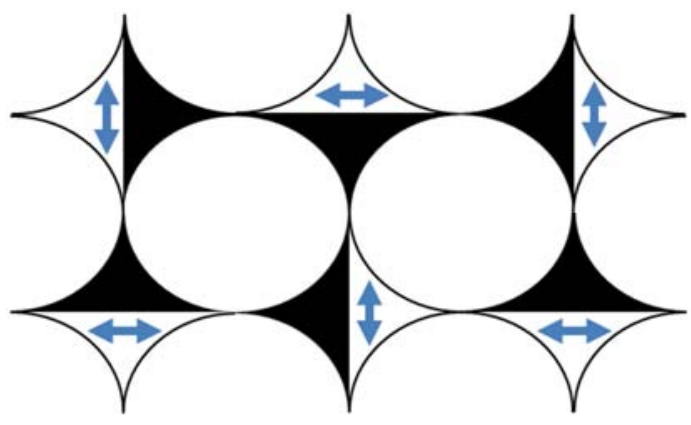

Figure 11. Vacuum polarization affecting spin interactions has been reported.

- Gravitational constant $\left(\mathrm{G}=6,67408 \times 10^{-11} \mathrm{~m}^{3} \mathrm{~kg}^{-1} \mathrm{~s}^{-2}\right)$ and its problem to measure with high accuracy since it can be related to the density exposed.

- Schrödinger equation, to describe how the quantum state of a quantum system changes with time, similar to Newton's second law.

- Planck's length $\left(1,616229 \times 10^{-35} \mathrm{~m}\right)$ which can indicate the distance between strings.

- Gluon size and its larger size far from earth.

- Black holes.

- ...

\section{Considerations For The Study}

Accelerate matter using a cyclotron to transform into energy so much matter as possible. We need a material with maximum magnetic permeability on high magnetic fields as possible, pure iron can be a good reference but we can consider some other materials with high permeability.

Particles reach their maximum energy at the periphery of the disc $(\mathrm{v}=\mathrm{qBR} / \mathrm{m})$, the output energy can be calculated when a speed is reached even in a relativistic approximation.

$$
E=1 / 2 m v^{2}=q 2 B 2 R 2 / 2 m
$$

Centripetal force, forces matter to the sides so we need a magnetic field to keep dimensions. We need enough width to study how strings bend and enough height to concentrate energy along (it is very difficult to concentrate kinetic energy at one point).

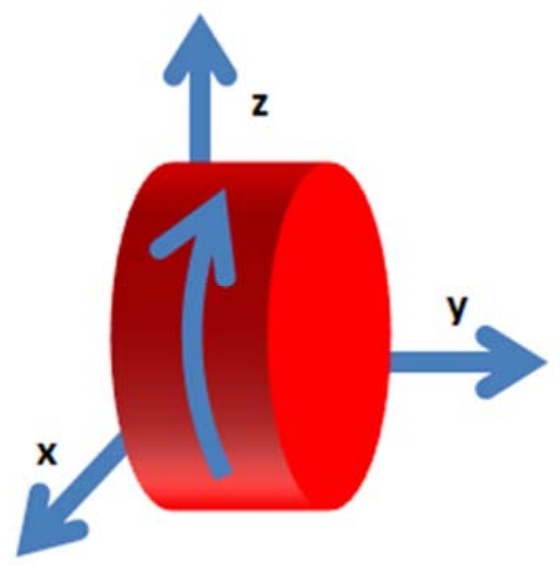

Figure 12. Accelerate cylinder particles until 1/10 speed of light. 
Calculate the energy of one disc in movement, a radius of $2 \mathrm{~cm}$ and a height of $2 \mathrm{~cm}$ are enough to study.

$$
\begin{aligned}
& V=\pi \times r^{2} \times h \\
& V=3,1416 \times 4 \times 2 \\
& V=25,1328 \mathrm{~cm}^{3}
\end{aligned}
$$

Calculate the mass using iron density $\left(\rho=7,874 \mathrm{gr} / \mathrm{cm}^{3}\right)$ and consider a maximum speed reached.

$$
\begin{gathered}
m=25,1328 \times 7,874=197,89 \mathrm{gr}=0,197 \mathrm{~kg} \\
v=300.000 \mathrm{~km} / \mathrm{sg}=3 \times 10^{7} \mathrm{~m} / \mathrm{sg}
\end{gathered}
$$

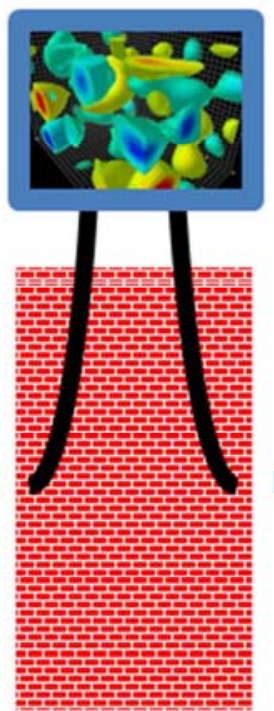

\section{Vacuum density changes}

\section{Energy density can be increased about $1 / 100$ at a speed of $3 \times 10^{7} \mathrm{~m} / \mathrm{sg}$}

Compare kinetic energy reached with the maximum energy which could be generated using a relativistic approximation.

$$
\begin{gathered}
E c=1 / 2 m v^{2} \\
E c=1 / 20,197 \times 9 \times 10^{14} \\
E c=0,8865 \times 10^{14} \\
E=m c^{2} \\
E=0,197 \times 9 \times 10^{16} \\
E=1,773 \times 10^{16}
\end{gathered}
$$

Figure 13. Energy avoid strings to penetrate. We need all energy at vertical to obtain significant values.

\section{References}

[1] Joseph Conlon (2015). Why String Theory?. CRC Press. ISBN 978-1-4822-4247-8. http://dx.doi.org/10.1063/PT.3.3201.

[2] Henning Genz (1999). Nothingness The Science of Empty Space. Addison-Wesley. ISBN 978-0201442335.

[3] Bechler, Z. (2012). Newton's Physics and the Conceptual Structure of the Scientific Revolution. Springer Science \& Business Media. http://dx.doi.org/10.1007/978-94-011-3276-3.

[4] Gould, P. H. (2013). Introduction to Linear Elasticity. http://dx.doi.org/10.1007/978-1-4614-4833-4.

[5] Cannon, J. T., \& Dostrovsky, S. (1975). The Evolution of Dynamics: Vibration Theory from 1687 to 1742. http://dx.doi.org/10.1007/978-1-4613-9461-7.

[6] F. Yndurain (1995). Limits on the mass of the gluon. Physics Letters B. 345 (4): 524. http://dx.doi.org/10.1016/03702693(94)01677-5.
[7] Williams, A. G., Bonnet, F. D., Bowman, P. O., Leinweber, D. B., Skullerud, J. I., \& Zanotti, J. M. (2002). The Transition from Nonperturbative to Perturbative QCD. In NonPerturbative QCD (pp. 189-196). http://dx.doi.org/10.1142/9789812778352 0026.

[8] J. Li, H. Y. Guo, B. N. Wan, X. Z. Gong, Y. F. Liang, G. S. $\mathrm{Xu}, \mathrm{K}$. F. Gan, J. S. Hu, H. Q. Wang, L. Wang, L. Zeng, Y. P. Zhao, P. Denner, G. L. Jackson, A. Loarte, R. Maingi, J. E. Menard, M. Rack \& X. L. Zou. A long-pulse high-confinement plasma regime in the Experimental Advanced Superconducting Tokamak. Nature Physics 9, 817-821 (2013). http://dx.doi.org/10.1038/nphys2795.

[9] Andreas S. Kronfeld \& Chris Quigg (2010). Resource Letter QCD-1: Quantum chromodynamics. http://dx.doi.org/10.1119/1.3454865.

[10] Wang, ZY. Plasmonics (2016) 11: 503. Modern Theory for Electromagnetic Metamaterials.

http://dx.doi.org/10.1007/s11468-015-0071-7. 